\title{
BMJ
}

\section{Neuraminidase inhibitors for preventing and treating influenza in healthy adults: systematic review and meta- analysis}

\author{
Tom Jefferson, researcher, ${ }^{1}$ Mark Jones, statistician, ${ }^{2}$ Peter Doshi, doctoral student, ${ }^{3}$ Chris Del Mar, dean; \\ coordinating editor of Cochrane Acute Respiratory Infections Group ${ }^{4}$
}

${ }^{1}$ Acute Respiratory Infections Group, Cochrane Collaboration,

Rome, Italy

2University of Queensland, School of Population Health, Brisbane, Australia

${ }^{3}$ Program in History Anthropology, Science,

Technology and Society,

Massachusetts Institute of

Technology, Cambridge, MA, USA

${ }^{4}$ Faculty of Health Sciences and Medicine, Bond University, Gold Coast, Australia

Correspondence to: C Del Mar cdelmar@bond.edu.au

Cite this as: BMJ 2009;339:b5106 doi:10.1136/bmj.b5106

\section{ABSTRACT}

Objectives To update a 2005 Cochrane review that assessed the effects of neuraminidase inhibitors in preventing or ameliorating the symptoms of influenza, the transmission of influenza, and complications from influenza in healthy adults, and to estimate the frequency of adverse effects.

Search strategy An updated search of the Cochrane central register of controlled trials (Cochrane Library 2009, issue 2), which contains the Acute Respiratory Infections Group's specialised register, Medline (1950Aug 2009), Embase (1980-Aug 2009), and postmarketing pharmacovigilance data and comparative safety cohorts.

Selection criteria Randomised placebo controlled studies of neuraminidase inhibitors in otherwise healthy adults exposed to naturally occurring influenza.

Main outcome measures Duration and incidence of symptoms; incidence of lower respiratory tract infections, or their proxies; and adverse events.

Data extraction Two reviewers applied inclusion criteria, assessed trial quality, and extracted data.

Data analysis Comparisons were structured into prophylaxis, treatment, and adverse events, with further subdivision by outcome and dose.

Results 20 trials were included: four on prophylaxis, 12 on treatment, and four on postexposure prophylaxis. For prophylaxis, neuraminidase inhibitors had no effect against influenza-like illness or asymptomatic influenza. The efficacy of oral oseltamivir against symptomatic laboratory confirmed influenza was $61 \%$ (risk ratio 0.39 , $95 \%$ confidence interval 0.18 to 0.85 ) at $75 \mathrm{mg}$ daily and $73 \%(0.27,0.11$ to 0.67$)$ at $150 \mathrm{mg}$ daily. Inhaled zanamivir $10 \mathrm{mg}$ daily was $62 \%$ efficacious $(0.38,0.17$ to 0.85). Oseltamivir for postexposure prophylaxis had an efficacy of $58 \%$ (95\% confidence interval $15 \%$ to $79 \%$ ) and $84 \%$ (49\% to $95 \%$ ) in two trials of households. Zanamivir performed similarly. The hazard ratios for time to alleviation of influenza-like illness symptoms were in favour of treatment: 1.20 (95\% confidence interval 1.06 to 1.35 ) for oseltamivir and 1.24 (1.13 to 1.36) for zanamivir. Eight unpublished studies on complications were ineligible and therefore excluded. The remaining evidence suggests oseltamivir did not reduce influenza related lower respiratory tract complications (risk ratio $0.55,95 \%$ confidence interval 0.22 to 1.35 ). From trial evidence, oseltamivir induced nausea (odds ratio 1.79 , $95 \%$ confidence interval 1.10 to 2.93 ). Evidence of rarer adverse events from pharmacovigilance was of poor quality or possibly under-reported.

Conclusion Neuraminidase inhibitors have modest effectiveness against the symptoms of influenza in otherwise healthy adults. The drugs are effective postexposure against laboratory confirmed influenza, but this is a small component of influenza-like illness, so for this outcome neuraminidase inhibitors are not effective. Neuraminidase inhibitors might be regarded as optional for reducing the symptoms of seasonal influenza. Paucity of good data has undermined previous findings for oseltamivir's prevention of complications from influenza. Independent randomised trials to resolve these uncertainties are needed.

\section{INTRODUCTION}

Neuraminidase inhibitors comprise nebulised zanamivir (Relenza; Glaxo Wellcome) and oral oseltamivir (Tamiflu; Gilead Sciences and F Hoffmann-La Roche), and others still under development for parenteral or long acting use. ${ }^{1}$ Inhibiting neuraminidasewhich, as with haemagglutin, is specific to influenzablocks the exit of the influenza virus from the host cell, thereby preventing replication in other than a few host cells. $^{2}$

The use of neuraminidase inhibitors has increased dramatically with the spread of the influenza A/H1N1 pandemic that began in April 2009, a novel and potentially serious infection. Partly because of the rise in resistance to amantadine and rimantadine and the lack of an effective vaccine, neuraminidase inhibitors became a widespread public health intervention. Their use for early containment and interruption was also recommended in many pandemic plans, and the World Health Organization had previously encouraged member countries to gain experience with them. $^{3}$ 


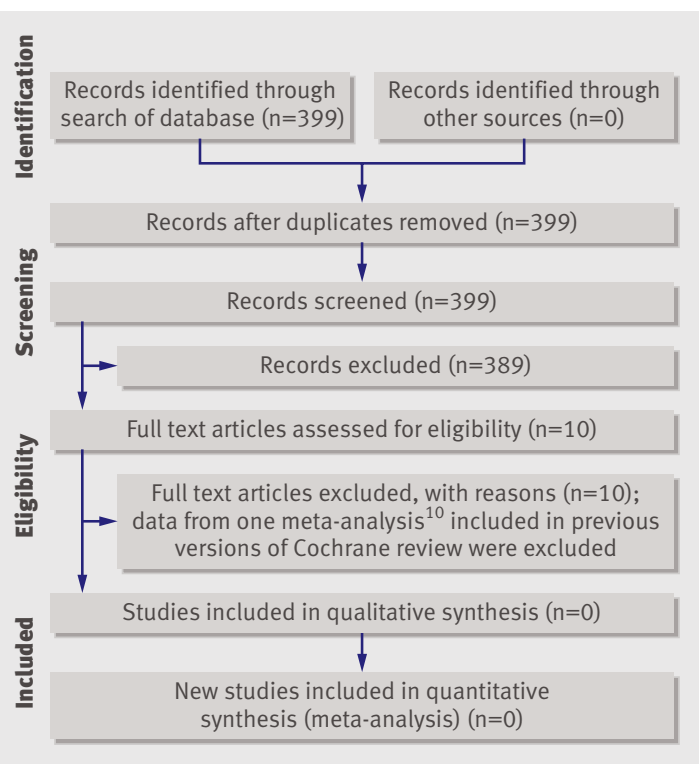

Fig 1| Flow of studies identified from 2009 update of randomised controlled trials

Although several systematic reviews of the effects of neuraminidase inhibitors are published, none systematically investigated the potential harms of the drugs. ${ }^{4-9}$ In addition, our previous Cochrane review ${ }^{6}$ summary of the evidence on the effects of oseltamivir on lower respiratory tract complications was criticised by Hayashi through the public Cochrane reviews feedback mechanism (see web extra on bmj.com). This criticism centred on one paper in particular, a meta-analysis of the effects of oseltamivir on complications of influenza. ${ }^{10}$ Only two of 10 randomised sets of data had been published, and Hayashi was concerned that information was insufficient to assess methods, reliability, and applicability of the eight remaining datasets.

In updating our review we addressed these additional concerns while answering the original questions: what is the evidence on the effects of neuraminidase inhibitors in preventing or ameliorating influenza, transmission of the virus, and influenza related complications in otherwise healthy adults, and what is the frequency of adverse effects? Our original review had found positive evidence on all of these effects, and gastrointestinal harms.

\section{METHODS}

We updated a search previously undertaken in any language for randomised or quasirandomised studies that compared oseltamivir or zanamivir in otherwise healthy people exposed to naturally occurring influenza, against placebo, control antivirals, or no intervention (or compared doses or schedules of the neuraminidase inhibitors) with the outcomes of influenza (efficacy) or influenza-like illness (effectiveness). ${ }^{6}$ We excluded experimental influenza challenge studies as their generalisability and comparability with field studies is uncertain. Studies had to include $75 \%$ or more of patients aged 14-60 (excluding older people at higher risk of complications). The updated search is summarised in the web extra. It included checking the references of other systematic reviews. ${ }^{47-9}$

Two of us separately read all titles and studies retrieved in the search and applied inclusion criteria. Disagreements were resolved by discussion with a third reviewer. Data were extracted on to standard forms, checked, and recorded. Assessing for risk of bias (using established criteria) ${ }^{11}$ became a major focus of this review.

Among complications we included a combined outcome of pneumonia, bronchitis, sinusitis, and otitis media requiring antibiotics in people with laboratory confirmed influenza. We were unable to meta-analyse the same outcomes reported by Kaiser et $\mathrm{al}^{10}$ because the data for those outcomes were not available to us for individual trials. We carried out a sensitivity analysis of complications by excluding the unpublished trials included in the Kaiser review criticised by Hayashi.

We used random effects methods to compare dichotomous outcomes (risk ratio for efficacy and odds ratio for safety); therefore estimates meta-analysed over multiple trials are average treatment effects. Where hazard ratios were not provided we converted the ratio of medians of treatment groups into (log) hazard ratios (estimating the variance of these) ${ }^{12}$ to enable meta-analysis of time to event outcomes.

We carried out an additional search for evidence of harms, including submitting a Freedom of Information Act request to the US Food and Drug Administration for all data on the harms of oseltamivir and zanamivir. ${ }^{13}$ We also pursued authors of some papers and manufacturers in attempts to disentangle summarised or conflated data.

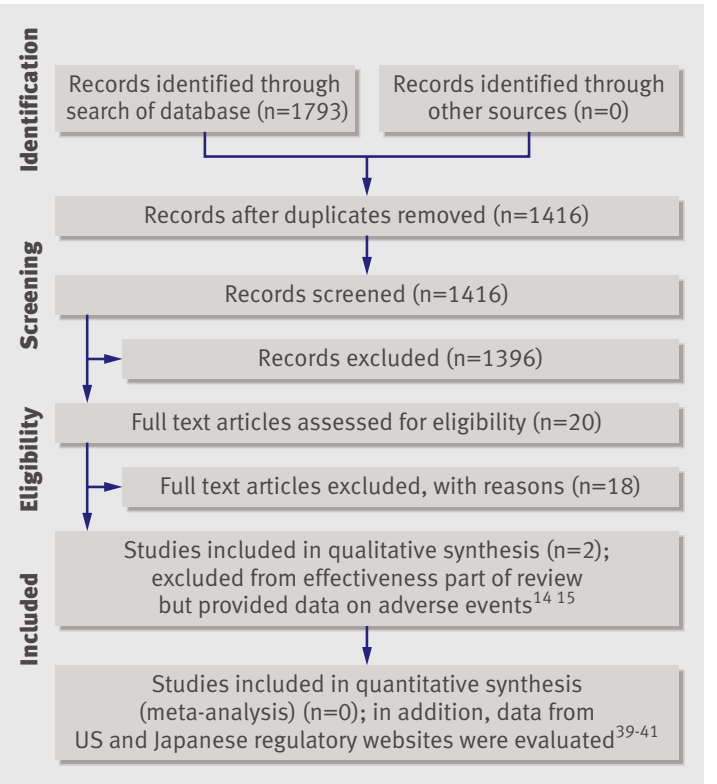

Fig 2 | Flow of studies identified from search for evidence from post-marketing studies (excluding adverse event reporting system) 


\section{RESULTS}

Overall, 29 studies made up of 10 effectiveness and 10 safety studies (six were identified by both searches) were excluded, together with a further three effectiveness studies, excluded after more deliberation (figs 1 and 2). ${ }^{101415}$ This left 20 included trials in 19 publications. ${ }^{16-34}$ Two studies that were excluded from the effectiveness outcome question were included in the safety data sources. ${ }^{145}$ (See web extra for details of the included and excluded studies.)

\section{Quality of evidence}

On the basis of the published text only five trials were judged adequate by usual Cochrane Collaboration methods $^{35}$ : one trial on prophylaxis ${ }^{30}$ and four on treatment. ${ }^{25273133}$ Most of the trials were at risk of

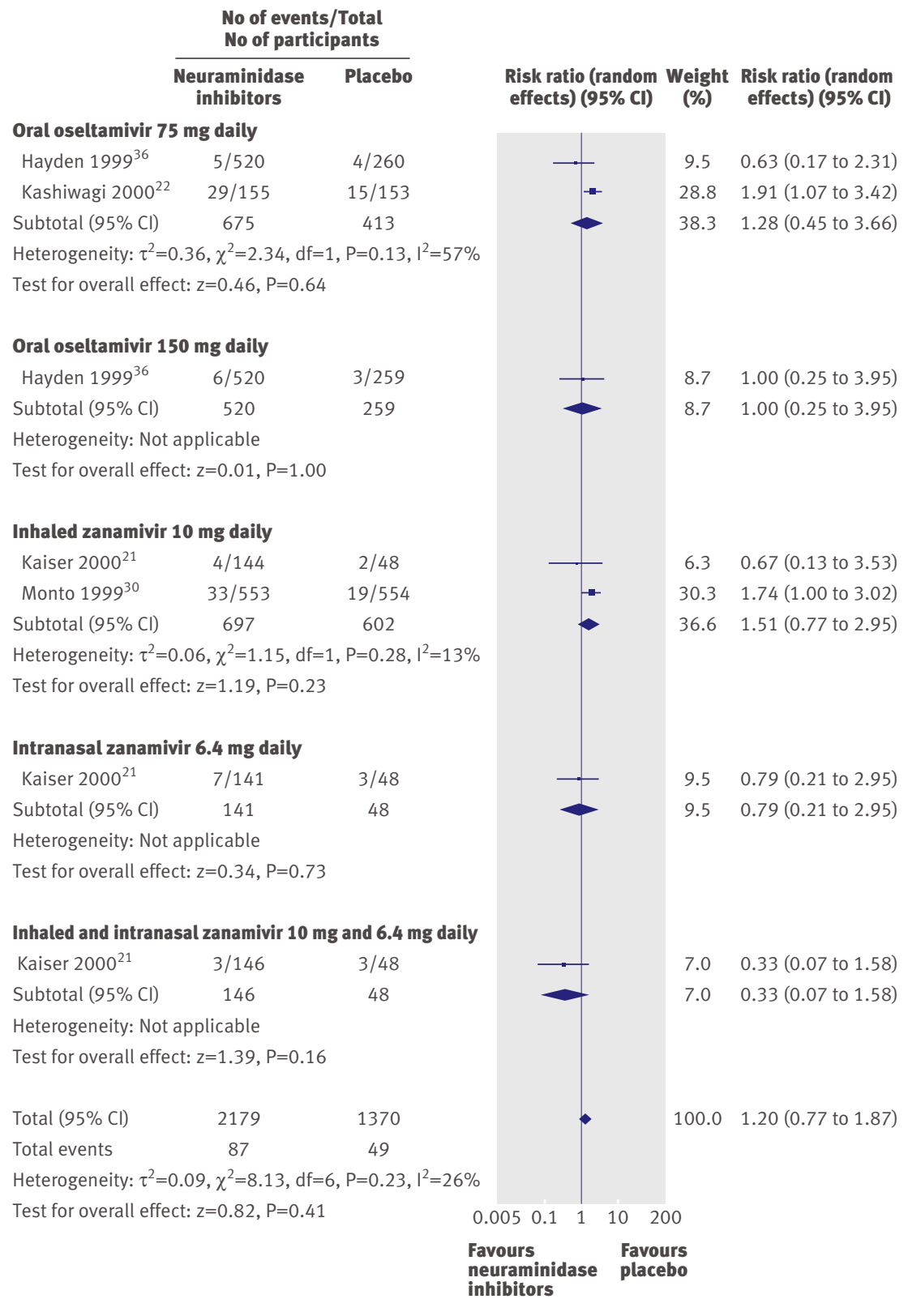

Fig 3 Effect of neuraminidase inhibitors compared with placebo on prophylaxis against influenza-like illness bias, arising from poor descriptions of the methods, ${ }^{10161721-2336}$ such as no description of losses to follow-up and blinding. ${ }^{21}$ Attempts to deal with these shortcomings were unsuccessful: although three of five first authors of studies on oseltamivir treatment responded to our contact, none had original data and referred us to the manufacturer (Roche), which was not able to unconditionally provide the information as quickly as we needed it to update this review. ${ }^{37}$ One meta-analysis ${ }^{10}$ was made up of data from 10 studies. We were obliged to exclude this meta-analysis because we were unable to determine the number of healthy adults experiencing complications in each study (some studies contained mixed populations of healthy and comorbid participants), nor the number of patients experiencing one or more of "bronchitis, lower respiratory tract infection, or pneumonia" presenting to each study.

\section{Evidence of benefits}

\section{Prophylaxis against influenza}

We found no new studies of benefit fulfilling our criteria. Two trials compared a total of 697 adults treated with inhaled zanamivir $10 \mathrm{mg}$ daily and 602 with placebo (followed for 22 days), ${ }^{2130}$ and two trials compared 675 adults treated with oral oseltamivir $75 \mathrm{mg}$ daily and 413 with placebo (followed for 49 days). ${ }^{2236}$ Evidence was insufficient to support or refute the effect of neuraminidase inhibitors on prophylaxis of influenza-like illness: risk ratio 1.28 (95\% confidence interval 0.45 to 3.66 ) for oseltamivir and 1.51 (0.77 to 2.95) for zanamivir. ${ }^{192136}$ Higher dosages made no difference, although this conclusion is based on a single study with only nine events (fig 3). ${ }^{36}$

Zanamivir reduced the chance of symptomatic laboratory confirmed influenza $(0.38,0.17$ to 0.85 for $10 \mathrm{mg}$ daily). Oseltamivir was similarly efficacious $(0.39,0.18$ to 0.85 for $75 \mathrm{mg}$ daily; fig 4$)$. Neither protected against asymptomatic influenza. ${ }^{223036}$

\section{Postexposure prophylaxis against influenza}

Postexposure prophylaxis against influenza entails giving exposed people neuraminidase inhibitors before symptoms develop. Two zanamivir trials reported significant protection for households (risk ratios $0.19^{30}$ and $\left.0.2^{19}\right)$ and two oseltamivir trials reported similar results $\left(0.16^{34}\right.$ and $\left.0.42^{18}\right)$.

\section{Treatment}

Eight trials of zanamivir treatment were included in the current review, ${ }^{16172025-2832}$ of which two ${ }^{1617}$ were linked to others ${ }^{2728}$ (a total of 1878 participants in the treatment arm and 1310 controls, with a mean length of follow-up of 26 days). Five trials of oseltamivir were also included, ${ }^{1024313338}$ on $^{10}$ with supplementary outcome data from previous trials and another ${ }^{24}$ linked to a redundant publication ${ }^{38}$ (totalling 1118 participants in the treatment arm and 679 controls, with a mean length of follow-up of 21 days).

There was evidence of benefit in shortening the duration of influenza-like illness for zanamivir (hazard 


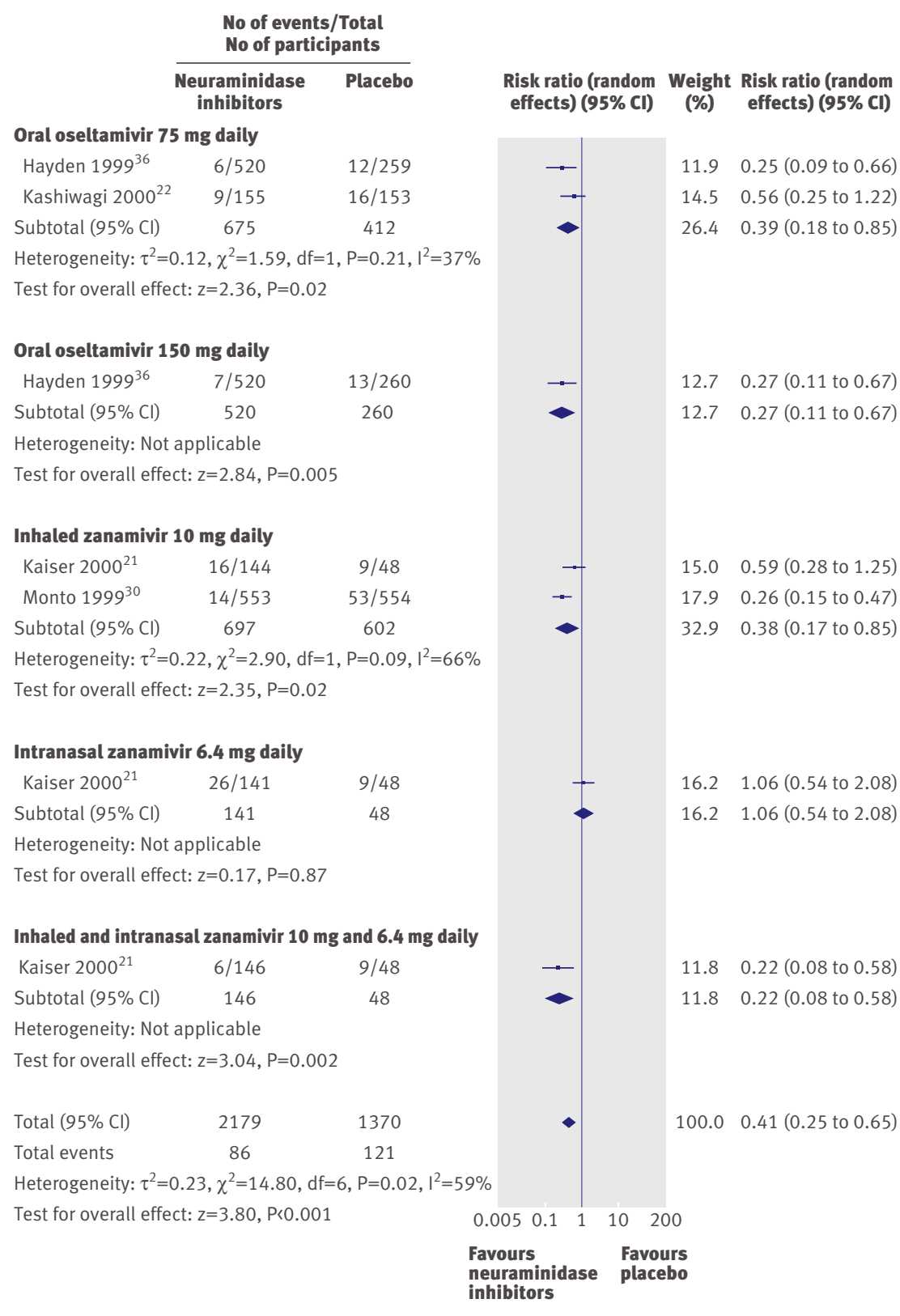

Fig 4 | Effect of neuraminidase inhibitors compared with placebo on prophylaxis against laboratory confirmed influenza provided information on the harms of oseltamivir. ${ }^{1415}$ Eighteen safety studies were included. This left 20 included trials in 19 publications. ${ }^{16-34}$ We also examined two sources of pharmacovigilance data describing reported adverse events from the FDA adverse event reporting system (AERS), one obtained by request using the Freedom of Information Act, "AERS-1", and another accessed directly from two FDA websites, "AERS-2." 3940 Another dataset containing reports of adverse events was located on a website by the Japanese Pharmaceuticals and Medical Devices Agency. ${ }^{41}$ We were unable to gain the same access to data from the European Medicines Agency. ${ }^{42}$ The table summarises the characteristics of the data sources.

\section{Quality of evidence}

In total, 1416 titles were found, most for oseltamivir, and we discarded all except for 20 studies of different design, the two sources of pharmacovigilance data from the AERS, and the Japanese data. ${ }^{1339-41}$ The Japanese data contained tables of adverse events occurring in manufacturer funded clinical trials, and included individual patient level data.

Both FDA datasets include post-licensure adverse event reports (voluntary and mandatory) from a variety of sources, including consumers, healthcare providers, and manufacturer. Reports in the FDA AERS database are of three types: "expedited (15-day)" and "periodic," which are mandatory reports from manufacturers, and "direct," which are reports voluntarily submitted by non-manufacturers. The two manufacturer channels comprised 2062/2275 (90.6\%) of all reports on oseltamivir from the AERS. All adverse events that are "both serious and unexpected" must, according to FDA rules, be reported by manufacturers within 15 days of initial receipt of information about the event. "Serious," by the FDA's definition, are those events that result in "Death, a life-threatening adverse drug experience, inpatient hospitalization or prolongation of existing hospitalization, a persistent or significant disability/incapacity, or a congenital anomaly/birth defect." ${ }^{, 43}$ For all other adverse events, reporting requirements differ on the basis of the origin of the information. Not all notices of adverse events received by the FDA are necessarily entered into the AERS.

The Japanese data contained tables of adverse events, including individual patient level data for serious adverse events. ${ }^{41}$ However, these were discarded because of irreconcilable differences in serious adverse events reported here but apparently not reported in two published trials. ${ }^{3133}$

\section{Evidence of harms}

The trials identified only one serious adverse event ${ }^{3}$ (so labelled in the Japanese data, a patient with neutropenia), and, in particular, no neuropsychiatric events. Oseltamivir induced nausea (odds ratio 1.79, 95\% confidence interval 1.10 to 2.93 ), especially at the higher dose of $150 \mathrm{mg}$ daily $(2.29,1.34$ to 3.92 ; fig 7$)$. No

\section{Evidence on harms of neuraminidase inhibitors}

Two of the studies excluded for addressing the benefits of neuraminidase inhibitors question nevertheless 


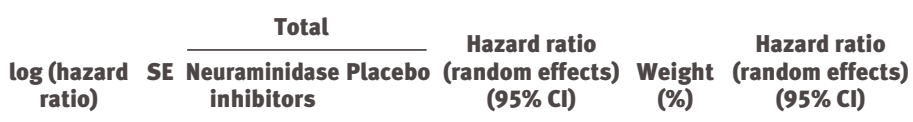

Zanamivir

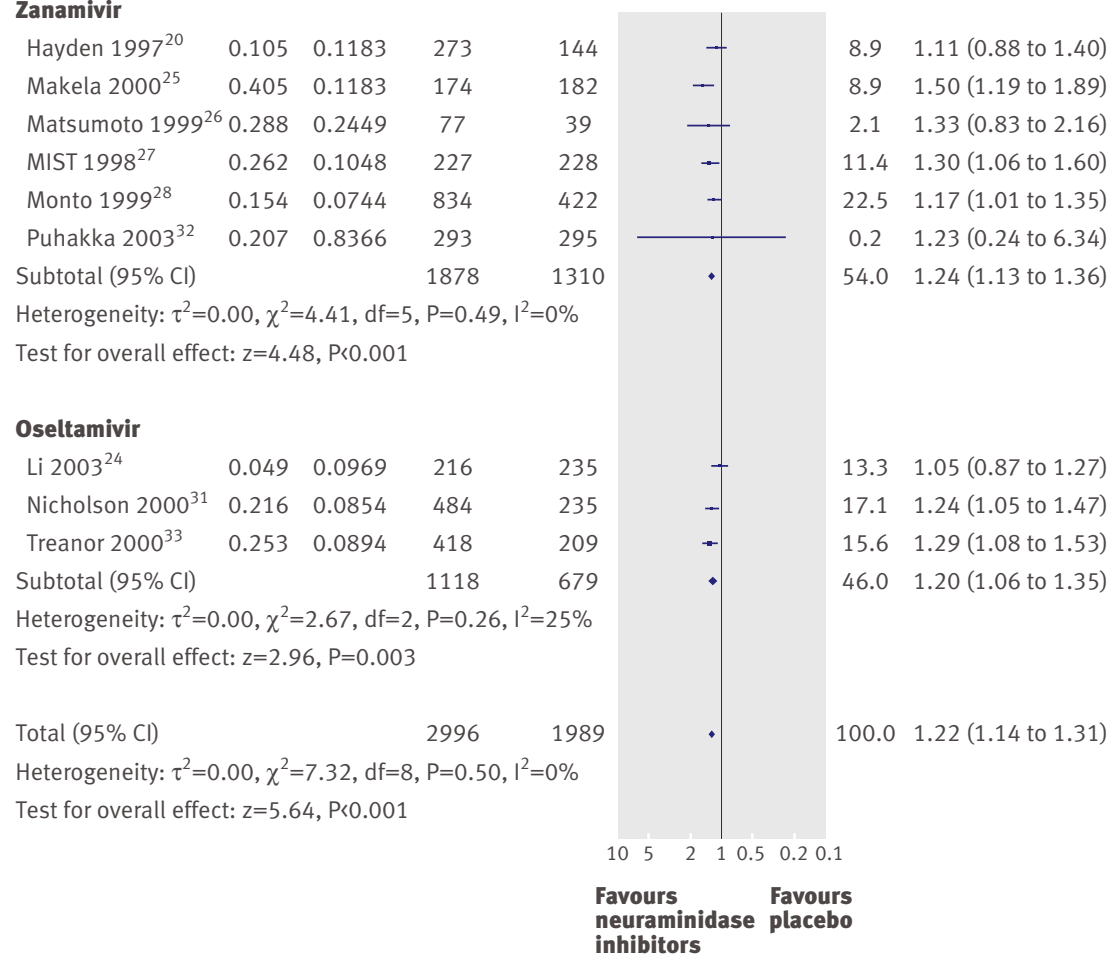

Fig 5 | Effect of neuraminidase inhibitors compared with placebo on alleviation of influenza symptoms (intention to treat analysis)

statistically significant adverse event was found for zanamivir from trials. ${ }^{26-2832}$

Two published studies reported additional retrospective comparative safety data on oseltamivir. ${ }^{1415}$
Their data suggest an incidence of neuropsychiatric adverse events per 1000 adults aged 18-49 of 20-27 at 14 days and 30-40 at 30 days, ${ }^{14}$ and for neuropsychiatric adverse events in prospective clinical trials, an incidence of $0.5 \% .^{15}$

AERS-1includes 2275 adverse event reports for oseltamivir and 453 for zanamivir (excluding followup reports on the same individual event) generated worldwide between December 1999 and July 2009 (the month our request was answered). Unfortunately it indicates neither reporting country nor how long the event occurred before receipt of the report by the FDA. The period from 2004 onwards overlaps with AERS-2, which has reports from January 2004 to March 2009, indicating both initial and follow-up reports, and reporting the date of the adverse event. ${ }^{3940}$ From July 2005 it indicates the reporting country. From July 2005 to March 2009, 1205 initial adverse events occurred. Most $(681,56.5 \%)$ were reported from Japan, followed by the United States (390, 32.4\%). Most (1109, 92.0\%) were for oseltamivir (perhaps reflecting its higher use). A disproportionate amount of reports are for people aged less than 20 (with data on age missing for many).

\section{DISCUSSION}

The data suggest that neuraminidase inhibitors are effective at reducing the symptoms of influenza. The evidence is of modest benefit-reduction of illness by about one day. This benefit has been generalised to assume benefits for very ill people in hospital. This seems reasonable, although it is worth remembering that we have no data to support this, and it is unlikely that ethics committees would allow a trial of no treatment for people with influenza who have life threatening disease.

\section{Summary of databases on adverse effects of neuraminidase inhibitors}

\section{Data source}

Who reports

US FDA AERS-1 (obtained through Freedom of

Information Act request)
Manufacturers under mandatory reporting requirements in accordance with FDA Code of Federal Regulations title 21 section 314.80 ; health professionals and consumers report voluntarily

\section{What is reported}

Post-marketing surveillance adverse event reports occurring in US and abroad. For adverse events occurring in US, manufacturers must report to FDA all adverse events except for labelled non-serious events (assuming companies have been granted such a waiver). For non-US events, manufacturers are only required to report adverse events that are "both serious and unexpected." For first three years after approval, FDA enters all adverse events into AERS database. After three years, all electronically submitted and all "serious" adverse events are entered into AERS

Post-marketing surveillance adverse event reports occurring Jan 2004 to Mar 2009 in US and abroad. For adverse events occurring in US, manufacturers must report to FDA all adverse events except for labelled non-serious events (assuming companies have been granted such a waiver). For non-US events, manufacturers are only required to report adverse events that are "both serious and unexpected." For first three years after approval, FDA enters all adverse events into AERS database. After three years, all electronically submitted and

all "serious" adverse events are entered into AERS

Japanese Pharmaceuticals and Medical Devices Agency $^{41}$
Manufacturers under mandatory reporting requirements in accorda with FDA professionals and consumers report voluntarily
Jul 2009
Documents reviewed were submitted by Chugai Pharmaceutical to Japanese regulators in conjunction with new drug application for prophylactic use of Tamiflu $75 \mathrm{mg}$ capsules numbers WV15670, WV15671, and WV15730. Individual patient level description of serious adverse events occurring events occurred between during these three trial protocols while receiving treatment Dec 1997 and Sep 1998 (six participants), not receiving treatment (two participants), and resulting in early withdrawal (12 participants)

\section{Date range of contents}

Aug 1999 (zanamivir) and Date of event, timing in Dec 1999 (oseltamivir) to relation to drug, country of report unclear

.

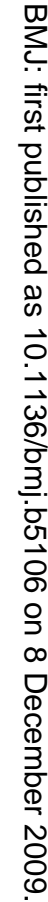

More detailed reports indicating reporting country consistently from Jul 2005 Reporting of neuropsychiatric adverse events more common among children and adolescents taking oseltamivir, but relation to drug is unclear ${ }^{48}$

No cases of neuropsychiatric adverse events were reported in trials of otherwise healthy adults 


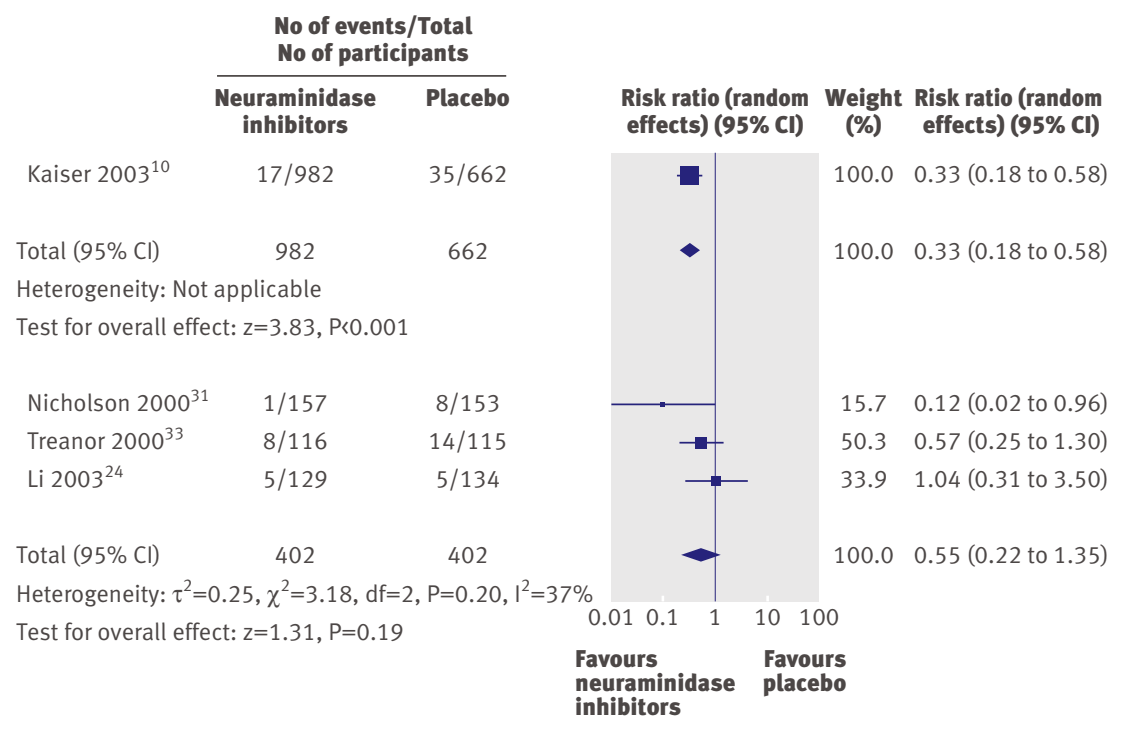

Fig 6 | Effect of oseltamivir compared with placebo on complications (including pneumonia, bronchitis, or "other lower respiratory tract infections") requiring antibiotics in laboratory confirmed influenza, based on study by Kaiser et $\mathrm{al}^{10}$ and three other studies (complications included pneumonia, bronchitis, otitis media, and sinusitis). ${ }^{243133}$ Unpublished studies were excluded
One important caveat to these results arises from concerns about the difference between efficacy (treatment response to pure influenza virus infection) and effectiveness (the real life response to influenza-like illness, when real cases of influenza are indistinguishable from other causative agents not responsive to neuraminidase inhibitors $\left.{ }^{44}\right)$. Understanding the proportion of influenza-like illness caused by both seasonal and epidemic influenza is critical to generalising the results of this review to clinical practice. The finding of treatment effectiveness for the neuraminidase inhibitors may have been enhanced by the high percentage of influenza-like illness caused by influenza in most of the included trials - for example, up to $80 \% .^{23}$

Data on the effectiveness of oseltamivir against the complications of influenza are confusing. Hayashi

\begin{tabular}{|c|c|c|c|c|c|c|}
\hline & \multicolumn{2}{|c|}{$\begin{array}{l}\text { No of events/Total } \\
\text { No of participants }\end{array}$} & \multirow{2}{*}{\multicolumn{2}{|c|}{$\begin{array}{l}\text { Odds ratio (random } \\
\text { effects) }(95 \% \mathrm{Cl})\end{array}$}} & \multirow[b]{2}{*}{$\begin{array}{c}\text { Weight } \\
\text { (\%) }\end{array}$} & \multirow[b]{2}{*}{$\begin{array}{l}\text { Odds ratio (random } \\
\text { effects) }(95 \% \mathrm{Cl})\end{array}$} \\
\hline & $\begin{array}{l}\text { Neuraminidase } \\
\text { inhibitors }\end{array}$ & Placebo & & & & \\
\hline \multicolumn{7}{|c|}{ Oral oseltamivir $75 \mathrm{mg}$ daily } \\
\hline Hayden $1999^{36}$ & $63 / 520$ & $19 / 260$ & $\Rightarrow$ & + & 83.8 & $1.75(1.02$ to 2.99$)$ \\
\hline Kashiwagi $2000^{22}$ & $8 / 155$ & $4 / 153$ & & 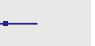 & 16.2 & 2.03 (0.60 to 6.88$)$ \\
\hline Subtotal $(95 \% \mathrm{Cl})$ & 675 & 413 & - & - & 100.0 & 1.79 (1.10 to 2.93$)$ \\
\hline \multicolumn{7}{|c|}{ Heterogeneity: $\tau^{2}=0.00, \chi^{2}=0.05, d f=1, P=0.83, I^{2}=0 \%$} \\
\hline \multicolumn{7}{|c|}{ Test for overall effect: $z=2.33, P=0.02$} \\
\hline \multicolumn{7}{|c|}{ Oral oseltamivir $150 \mathrm{mg}$ daily } \\
\hline Hayden $1999^{36}$ & $76 / 520$ & $18 / 259$ & & + & 100.0 & 2.29 (1.34 to 3.92$)$ \\
\hline Subtotal $(95 \% \mathrm{Cl})$ & 520 & 259 & & 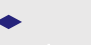 & 100.0 & 2.29 (1.34 to 3.92$)$ \\
\hline \multicolumn{3}{|c|}{ Heterogeneity: Not applicable } & $0.020 .1 \quad 1$ & 105 & & \\
\hline \multicolumn{3}{|c|}{ Test for overall effect: $z=3.03, P=0.002$} & \multicolumn{4}{|c|}{$\begin{array}{ll}\text { Favours } & \text { Favours } \\
\text { neuraminidase } \\
\text { inhibitors }\end{array}$} \\
\hline
\end{tabular}

Fig 7| Effect of oseltamivir compared with placebo on nausea (intention to treat analysis) pointed out that the original data, which led to the 2005 version of this original Cochrane review reporting benefits for oseltamivir on reduction of complications from lower respiratory tract infection, principally came from one meta-analysis that summarised 10 trials containing a mixture of published and unpublished data. ${ }^{10}$ Only two of the trials it contains are published (and are reported in this Cochrane review update), the remainder were offered to us under conditions we thought unacceptable, and what was offered to us was insufficient to analyse properly. (Comments on the Kaiser et al paper are in the web extra). This means we are now obliged to exclude the meta-analysis. ${ }^{10}$ The remaining published evidence is insufficient to answer the question about the effectiveness of either neuraminidase inhibitor on reducing the complications of lower respiratory tract infection, antibiotic use, or admissions to hospital. It is possible that there is a publication bias, especially as we know of eight trials that are unpublished and inaccessible. We have not undertaken a funnel plot because there are only three trials (fig 6), and so the issue of publication bias remains unresolved. Its direction might be in favour of exaggerating the treatment effect. Hayashi's comments point out a serious problem with our original review, which we now address.

The results from the meta-analyses involving hazard ratios should be viewed with caution because of the approximate methods used to extract estimates for each study. Hazard ratios were rarely reported directly, so we had to use the ratio of the observed median duration of symptoms in each group as an approximation to the hazard ratio. This approach may be overly simplistic, as it makes a comparison at only one time point and assumes a constant survival difference over time, and thus may produce different metaanalysis results than if the actual hazard ratio estimates were available. ${ }^{45}$

Role of neuraminidase inhibitors in seasonal influenza Neuraminidase inhibitors had low effectiveness and high efficacy against symptoms (shortening the illness by half to one day, a crude estimate made by applying a hazard ratio of 1.2 on the control length of illness of four days to about 3.3 days, a reduction of less than one day, and preventing symptoms from appearing), and initially seemed to be well tolerated (with the possible exception of oseltamivir induced nausea and vomiting).

A surprising finding was the high percentage (57$80 \%)^{23}$ of influenza in the trial populations receiving neuraminidase inhibitors. We remain at a loss to explain this because most other data suggest much lower rates. ${ }^{46}$

Role of neuraminidase inhibitors in pandemic influenza We identified no direct comparative evidence of the role of neuraminidase inhibitors in avian influenza $\mathrm{A} / \mathrm{H} 5 \mathrm{~N} 1$ or in the current novel influenza $\mathrm{A} / \mathrm{H} 1 \mathrm{~N} 1$ pandemic. This means that we have to generalise from the trials, and this seems reasonable given that 


\section{WHAT IS ALREADY KNOWN ON THIS TOPIC}

Neuraminidase inhibitors (especially oseltamivir) have become global public health drugs for influenza

They prevent symptoms and shorten the duration of illness by about one day if taken within 48 hours of the onset of symptoms

Toxicity and the effects on complications have been debated

\section{WHAT THIS STUDY ADDS}

Neuraminidase inhibitors reduce the symptoms of influenza modestly

Neuraminidase inhibitors reduce the chance of people exposed to influenza developing laboratory confirmed influenza but not influenza-like illness

Evidence for or against their benefit for preventing complications of influenza is insufficient

Evidence for or against serious adverse events is lacking, although oseltamivir causes nausea

the pandemic influenza $\mathrm{A} / \mathrm{H} 1 \mathrm{~N} 1$ virus will likely be acted on in the same biological manner as previously circulating influenza viruses, such as seasonal A/H1N1.

Neuraminidase inhibitors do not, however, prevent infection or stop nasal viral excretion, so they may be a suboptimal means of interrupting viral spread in a pandemic. If used to contain a severe pandemic outbreak, neuraminidase inhibitors should be considered only part of a package of measures to interrupt spread, including physical measures. ${ }^{47}$

\section{Possible harms of neuraminidase inhibitors}

We focused on oseltamivir because of the considerably greater global experience with this drug. Post-marketing pharmacovigilance data about oseltamivir obtained from the FDA are of limited use because of likely under-representation of reports generated from outside the United States. The entire AERS database (containing adverse event reports of all types) between 1999 and 15 September 2007 contains only 1805 reports. However, the Roche global safety database contains reports of 2466 neuropsychiatric adverse events during this time, of which $562(22.8 \%)$ were classified as "serious."15

Another important limitation of the AERS database is the practice by the FDA of not registering non-electronically submitted reports of non-serious adverse events three years after a drug's initial approval (personal correspondence with FDA, 14 October 2009).

Our findings of a possible association with neuraminidase inhibitors and the onset of rare harms coming from the US AERS data ${ }^{48}$ accord with a review of phase IV evidence from eight cases (adolescents and adults) ${ }^{49}$ suggesting oseltamivir may induce sudden behavioural changes in recipients, including hallucination, suicidal tendencies, and sudden death while asleep. This evidence came soon after a review ordered by the Japanese government, in part triggered by the 567 serious neuropsychiatric cases received since the 2001 launch of the drug and May 2007..$^{49} \mathrm{It}$ is, however, estimated that more than 36 million doses have been prescribed since $2001,{ }^{15}$ making such harms (even if confirmed) rare.

We therefore found under-reported evidence of varied quality, which could not answer concerns about the toxicity of neuraminidase inhibitors, especially oseltamivir. Governments should set up studies to monitor the safety of neuraminidase inhibitors. ${ }^{48}$

\section{Conclusion}

Because of the moderate effectiveness of neuraminidase inhibitors, we believe they should not be used in routine control of seasonal influenza. We are unsure of the generalisability of our conclusions from seasonal to pandemic influenza. Evidence on the effects of oseltamivir in complications from lower respiratory tract infections, reported in our 2006 Cochrane review, may be unreliable. Evidence on serious harms of neuraminidase inhibitors is limited. Independent randomised trials to resolve the uncertainties surrounding effectiveness are needed.

We thank Liz Dooley, Dr Aoki, Jon Deeks, Carlo Di Pietrantonj, and Vittorio Demicheli for input and advice on earlier versions of this review; Liz Dooley, Janet Wale, John Bartlett, Sree Nair, Tom Fahey, Ruth Foxlee, and Alex Rivetti for help with the searches; and the Australian National Health and Medical Research Council and the UK NHS Research and Development fund for grants to enable this international rapid update. Contributors: TOJ reapplied inclusion criteria for the 2009 update. All authors reappraised and investigated extracted data, while CDM supervised the process and arbitrated when necessary. MJ and PD checked and transformed data and supervised the revised meta-analysis. TOJ, CDM, MJ, and PD edited the text. All authors contributed to the final draft. CDM and TOJ are the guarantors.

Funding: This study was supported by grants from the Australian National Health and Medical Research Council and the UK NHS Research and Development fund

Competing interests: All authors have completed the Unified Competing Interest form at www.icmje.org/coi_disclosure.pdf (available on request from the corresponding author) and declare that (1) CDM and TOJ have support from the UK National Institute for Health Research and the Australian National Health and Medical Research Council for the submitted work; (2) CDM has provided expert advice to GlaxoSmithKline about vaccination against acute otitis media; (3) their spouses, partners, or children have no financial relationships that may be relevant to the submitted work; and (4) no authors have non-financial interests that may be relevant to the submitted work.

Ethical approval: Not required.

1 Hayden F. Developing new antiviral agents for influenza treatment: what does the future hold? Clin Infect Dis 2009;48(suppl 1):S3-13.

2 Moscona A. Neuraminidase inhibitors for influenza. N Engl J Med 2005;353:1363-73.

3 World Health Organization. Global agenda on influenza surveillance and control. 2002. www.who.int/csr/disease/influenza/ csrinfluenzaglobalagenda/en/print.html.

4 Burls A, Clark W, Stewart T, Preston C, Bryan S, Jefferson T, et al. Zanamivir for the treatment of influenza in adults: a systematic review and economic evaluation. Health Technol Assess 2002;6:1-87.

5 Cooper NJ, Sutton AJ, Abrams KR, Wailoo A, Turner D, Nicholson KG. Effectiveness of neuraminidase inhibitors in treatment and prevention of influenza $A$ and $B$ : systematic review and metaanalyses of randomised controlled trials. BMJ 2003;326:1235

6 Jefferson TO, Demicheli V, Di Pietrantonj C, Jones M, Rivetti D. Neuraminidase inhibitors for preventing and treating influenza in healthy adults. Cochrane Database Syst Rev 2006; 3:CD001265.

7 Tappenden P, Jackson R, Cooper K, Rees A, Simpson E, Read R, et al. Amantadine, oseltamivir and zanamivir for the prophylaxis of influenza (including a review of existing guidance No 67): a systematic review and economic evaluation. Health Technol Assess 2009;13:iii, ix-xii, 1-246.

8 Turner D, Wailoo A, Nicholson K, Cooper N, Sutton A, Abrams K. Systematic review and economic decision modelling for the 
prevention and treatment of influenza A and B. Health Technol Assess 2003;7:iii-iv, xi-xiii, 1-170.

9 Burch J, Corbett M, Stock C, Nicholson K, Elliot AJ, Duffy S, et al. Prescription of anti-influenza drugs for healthy adults: a systematic review and meta-analysis. Lancet Infect Dis 2009;9:537-45.

10 Kaiser L, Wat C, Mills T, Mahoney P, Ward P, Hayden F. Impact of oseltamivir treatment on influenza-related lower respiratory tract complications and hospitalizations. Arch Intern Med 2003;163:1667-72.

11 Higgins JPT, Green S. Cochrane handbook for systematic reviews of interventions Version 5.0.1. The Cochrane Collaboration, 2008. Available from www.cochrane-handbook.org.

12 Parmar MK, Torri V, Stewart L. Extracting summary statistics to perform meta-analyses of the published literature for survival endpoints. Stat Med 1998;17:2815-34.

13 US Food and Drug Administration: adverse events reporting system. 2009. www.fda.gov/Drugs/

GuidanceComplianceRegulatoryInformation/Surveillance/ AdverseDrugEffects/default.htm.

14 Blumentals WA, Song X. The safety of oseltamivir in patients with influenza: analysis of healthcare claims data from six influenza seasons. MedGenMed 2007;9:23.

15 Toovey S, Rayner C, Prinssen E, Chu T, Donner B, Thakrar B, et al. Assessment of neuropsychiatric adverse events in influenza patients treated with oseltamivir: a comprehensive review. Drug Saf 2008;31:1097-114.

16 Aoki FY, Fleming DM, Griffin AD, Lacey LA, Edmundson S. Impact of zanamivir treatment on productivity, health status and healthcare resource use in patients with influenza. Zanamivir Study Group. Pharmacoeconomics 2000;17:187-95.

17 Boivin G, Goyette N, Hardy I, Aoki F, Wagner A, Trottier S. Rapid antiviral effect of inhaled zanamivir in the treatment of naturally occurring influenza in otherwise healthy adults. J Infect Dis 2000;181:1471-4.

18 Hayden FG, Belshe R, Villanueva C, Lanno R, Hughes C, Small I, et al. Management of influenza in households: a prospective, randomized comparison of oseltamivir treatment with or without postexposure prophylaxis. J Infect Dis 2004;189:440-9.

19 Hayden FG, Gubareva LV, Monto AS, Klein TC, Elliot MJ, Hammond JM, et al. Inhaled zanamivir for the prevention of influenza in families. Zanamivir Family Study Group. N Engl J Med 2000;343:1282-9.

20 Hayden FG, Osterhaus AD, Treanor JJ, Fleming DM, Aoki FY, Nicholson KG, et al. Efficacy and safety of the neuraminidase inhibitor zanamivir in the treatment of influenzavirus infections. GG167 Influenza Study Group. N Engl J Med 1997;337:874-80.

21 Kaiser L, Henry D, Flack NP, Keene O, Hayden FG. Short-term treatment with zanamivir to prevent influenza: results of a placebocontrolled study. Clin Infect Dis 2000;30:587-9.

22 Kashiwagi S, Kudoh S, Watanabe A, Yoshimura I. [Efficacy and safety of the selective oral neuraminidase inhibitor oseltamivir fo prophylaxis against influenza-placebo-controlled double-blind multicenter phase III trial]. Kansenshogaku Zasshi 2000;74:1062-76

23 Kashiwagi S, Kudoh S, Watanabe A, Yoshimura I. [Clinical efficacy and safety of the selective oral neuraminidase inhibitor oseltamivir in treating acute influenza-placebo-controlled double-blind multicenter phase III trial.] Kansenshogaku Zasshi 2000;74:1044-61.

24 Li L, Cai B, Wang M, Zhu Y. A double-blind, randomized, placebocontrolled multicenter study of oseltamivir phosphate for treatment of influenza infection in China. Chin Med J (Engl) 2003;116:44-8.

25 Makela MJ, Pauksens K, Rostila T, Fleming DM, Man CY, Keene ON, et al. Clinical efficacy and safety of the orally inhaled neuraminidase inhibitor zanamivir in the treatment of influenza: a randomized, double-blind, placebo-controlled European study. J Infect 2000;40:42-8.

26 Matsumoto K, Ogawa N, Nerome K, Numazaki Y, Kawakami Y, Shirato K, et al. Safety and efficacy of the neuraminidase inhibitor zanamivir in treating influenza virus infection in adults: results from Japan. GG167 Group. Antivir Ther 1999;4:61-8.

27 MIST Study Group. Randomised trial of efficacy and safety of inhaled zanamivir in treatment of influenza A and B virus infections. The MIST (Management of Influenza in the Southern Hemisphere Trialists) Study Group. Lancet 1998;352:1877-81.

28 Monto AS, Fleming DM, Henry D, de Groot R, Makela M, Klein T, et al. Efficacy and safety of the neuraminidase inhibitor zanamivir in the treatment of influenza A and B virus infections. J Infect Dis 1999;180:254-61.

29 Monto AS, Pichichero ME, Blanckenberg SJ, Ruuskanen O, Cooper C, Fleming DM, et al. Zanamivir prophylaxis: an effective strategy for the prevention of influenza types $A$ and $B$ within households. I Infect Dis 2002;186:1582-8.

30 Monto AS, Robinson DP, Herlocher ML, Hinson JM Jr, Elliott MJ, Crisp A. Zanamivir in the prevention of influenza among healthy adults: a randomized controlled trial. JAMA 1999;282:31-5.

31 Nicholson KG, Aoki FY, Osterhaus AD, Trottier S, Carewicz O, Mercier $\mathrm{CH}$, et al. Efficacy and safety of oseltamivir in treatment of acute influenza: a randomised controlled trial. Neuraminidase Inhibitor Flu Treatment Investigator Group. Lancet 2000;355:1845-50.

32 Puhakka T, Lehti H, Vainionpaa R, Jormanainen V, Pulkkinen M, Sharp S, et al. Zanamivir: a significant reduction in viral load during treatment in military conscripts with influenza. Scand I Infect Dis 2003;35:52-8.

33 Treanor JJ, Hayden FG, Vrooman PS, Barbarash R, Bettis R, Riff D, et al. Efficacy and safety of the oral neuraminidase inhibitor oseltamivir in treating acute influenza: a randomized controlled trial. US Oral Neuraminidase Study Group. JAMA 2000;283:1016-24.

34 Welliver R, Monto AS, Carewicz O, Schatteman E, Hassman M, Hedrick J, et al. Effectiveness of oseltamivir in preventing influenza in household contacts: a randomized controlled trial. JAMA 2001;285:748-54

35 Higgins JPT, Altman DG, for the Cochrane Statistical Methods Group and the Cochrane Bias Methods Group. Chapter 8: Assessing risk of bias in included studies. In: Higgins JPT, Green S, eds. Cochrane handbook for systematic reviews of interventions Version 5.0.2 [updated September 2009]. The Cochrane Collaboration, 2008. Available from www.cochrane-handbook.org.

36 Hayden FG, Atmar RL, Schilling M, Johnson C, Poretz D, Paar D, et al. Use of the selective oral neuraminidase inhibitor oseltamivir to prevent influenza. N Engl J Med 1999;341:1336-43.

37 Doshi P. Neuraminidase inhibitors-the story behind the Cochrane review. BMJ 2009;339:b5164.

38 Li L, Cai B, Wang M, Zhu Y. [A multicenter study of efficacy and safety of oseltamivir in treatment of naturally acquired influenza] Zhonghua Nei Ke Za Zhi 2001;40:838-42.

39 US Food and Drug Administration. The adverse event reporting system (AERS): older quarterly data files. 2009. www.fda.gov/Drugs/ GuidanceComplianceRegulatorylnformation/Surveillance/ AdverseDrugEffects/ucm083765.htm.

40 US Food and Drug Administration. The adverse event reporting system (AERS): latest quarterly data files. 2009. www.fda.gov/ Drugs/GuidanceComplianceRegulatoryInformation/Surveillance/ AdverseDrugEffects/ucm082193.htm.

41 Japan Pharmaceuticals and Medical Devices Agency. New drug approval related information. 2009. www.info.pmda.go.jp/ shinyaku/shinyaku hanbaimei index.html.

42 European Medicine Agency. Tamiflu. Annex 1: summary of product characteristics. p10, 2009. www.emea.europa.eu/humandocs/ PDFs/EPAR/tamiflu/emea-combined-h402en.pdf.

43 US Food and Drug Administration (FDA). CFR-Code of Federal Regulations Title 21 . Section 314.80 postmarketing reporting of adverse drug experiences. 2009. www.accessdata.fda.gov/scripts/ cdrh/cfdocs/cfcfr/CFRSearch.cfm?fr=314.80.

44 Smith J, Dutkowski R, Ward P. Antivirals for influenza in healthy adults. Lancet 2006;367:1571,1573; author reply.

45 Michiels S, Piedbois P, Burdett S, Nathalie S, Stewart L, Pignon JP. Meta-analysis when only the median survival times are known: a comparison with individual patient data results. Int J Technol Assess Health Care 2005;21:119-25.

46 Jefferson TO. Guest editorial. Mistaken identity: seasonal influenza versus influenza-like illness. Clin Evid 2009. http://clinicalevidence.bmj.com/downloads/05-10-09.pdf.

47 Jefferson T, Del Mar C, Dooley L, Ferroni E, Al-Ansary LA, Bawazeer GA, et al. Physical interventions to interrupt or reduce the spread of respiratory viruses: systematic review. BMJ 2009;339:b3675.

48 Jefferson T, Jones M, Doshi P, Del Mar C. Possible harms of oseltamivir-a call for urgent action. Lancet 2009;374:1312-3.

49 Hama R. Fatal neuropsychiatric adverse reactions to oseltamivir: case series and overview of causal relationships. Int J Risk Saf Med 2008;20:5-36

Accepted: 26 November 2009 